\title{
First National Anti-Tuberculosis Drug Resistance Survey (NDRS) from India - An Eye Opener
}

\author{
Gyanshankar P. Mishra ${ }^{1 *}$, Jasmin D. Mulani² \\ 'Department of Respiratory Medicine, Indira Gandhi Government Medical College, Nagpur, Maharashtra, India \\ ${ }^{2}$ Department of Biochemistry, Government Medical College, Nagpur, Maharashtra, India
}

\section{Article Info}

\section{Article Notes}

Received: August 07, 2018

Accepted: September 15, 2018

\section{*Correspondence:}

Dr. Gyanshankar P. Mishra, Associate Professor, Dept. of Respiratory Medicine, Indira Gandhi Government Medical College, C.A. Road, Nagpur, Maharashtra, India- 440018; Telehone No: 91-9850349074:

Email: gpmishra81@gmail.com

${ }^{\oplus} 2018$ Mishra GP. This article is distributed under the terms of the Creative Commons Attribution 4.0 International License.

\section{Keywords:}

Tuberculosis

TB

Drug Resistance

MDR TB

XDR TB

Drug-resistant TB

NDRS Survey

India

\section{Abstract}

Recently the report of the first National Anti-Tuberculosis Drug Resistance Survey (NDRS) from India was released on the occasion of World TB Day this year, i.e., 24th March 2018. The salient features were as follows: 1 . MDR-TB is $6.19 \%(\mathrm{Cl}$ 5.54-6.90\%) among all TB patients with $2.84 \%$ (Cl $2.27-3.50 \%$ ) among new and $11.60 \%$ (Cl 10.21-13.15\%) among previously treated TB patients. 2. Among MDR-TB patients, additional resistance to any fluoroquinolones was $21.82 \%(17.33-26.87 \%)$, and $3.58 \%(1.8-6.32 \%)$ to any second-line injectable drugs. 3. Among MDR-TB patients, additional resistance to at least one drug from each of the two classes, i.e., fluoroquinolone and second-line injectable drugs (XDR-TB) was 1.3\% (0.36-3.30\%). 4. Any first- or second line drug resistance among all TB patients is $28.0 \%$ (Cl 26.77- 29.29\%) with $22.54 \%$ ( $\mathrm{Cl} 21.10-24.10 \%$ ) among new and $36.82 \%$ (Cl 34.64-39.04\%) among previously treated TB patients. 5. Any isoniazid resistance is $11.06 \%$ (Cl 9.97-12.22\%) and $25.09 \%(\mathrm{Cl} 23.1--27.11 \%)$ among new and previously treated TB patients, respectively. 6 . Any pyrazinamide resistance is $6.95 \%(\mathrm{Cl}$ $6.07-7.91 \%)$ and $8.77 \%(7.53-10.13 \%)$ among new and previously treated TB patients, respectively. The current article reviews the findings of the survey along with their practical implications in the present-day clinical scenario.

\section{Introduction}

Recently the report of the first National Anti-Tuberculosis Drug Resistance Survey (NDRS) from India was released on the occasion of World TB Day this year, i.e., $24^{\text {th }}$ March $2018^{1}$. The survey represents the largest ever NDRS conducted by any country in the world and the first ever survey having drug susceptibility testing (DST) for 13 antiTB drugs using the automated liquid culture system, mycobacteria growth indicator tube (MGIT) 960. A total of 5280, sputum smearpositive pulmonary TB (Tuberculosis) patients (3240 new and 2040 previously treated) diagnosed at designated microscopy centres were enrolled in the survey. The salient results of the survey are shown in Table 1.

\section{Discussion}

The key finding of this study was the proportion of Multi-Drug Resistant TB (MDR-TB) among new and retreatment TB cases were $2.84 \%$ and $11.60 \%$ respectively. Monoresistance to rifampicin was not observed among new TB patients, thereby indicating that rifampicin resistance was always accompanied by isoniazid resistance. These figures are below the recently released global estimates of $3.6 \%$ of new cases and $17 \%$ of retreatment cases having MDR/RR (Rifampicin Resistance) $\mathrm{TB}^{2}$. A possible reason for this could be the non-inclusion of TB patients visiting private sector 
Table 1. Salient results of the first National Anti- Tuberculosis Drug Resistance Survey (NDRS) from India.

\begin{tabular}{|r|l|}
\hline Sr. No. & \multicolumn{1}{c|}{ Salient Results } \\
\hline 1 & $\begin{array}{l}\text { MDR-TB is 6.19\% (CI 5.54-6.90\%) among all TB patients with 2.84\% (Cl 2.27-3.50\%) among new and 11.60\% (CI 10.21-13.15\%) } \\
\text { among previously treated TB patients. }\end{array}$ \\
\hline 2 & $\begin{array}{l}\text { Among MDR-TB patients, additional resistance to any fluoroquinolones was 21.82\% (17.33-26.87\%), and 3.58\% (1.8-6.32\%) to } \\
\text { any second-line injectable drugs. }\end{array}$ \\
\hline 3 & $\begin{array}{l}\text { Among MDR-TB patients, additional resistance to at least one drug from each of the two classes, i.e. fluoroquinolone and sec- } \\
\text { ond-line injectable drugs (XDR-TB) was 1.3\% (0.36-3.30\%). }\end{array}$ \\
\hline 4 & $\begin{array}{l}\text { Any first- or second line drug resistance among all TB patients is 28.0\% (CI 26.77-29.29\%) with 22.54\% (Cl 21.10-24.10\%) among } \\
\text { new and 36.82\% (Cl 34.64-39.04\%) among previously treated TB patients. }\end{array}$ \\
\hline 5 & $\begin{array}{l}\text { Any isoniazid resistance is 11.06\% (Cl 9.97-12.22\%) and 25.09\% (Cl 23.1--27.11\%) among new and previously treated TB patients, } \\
\text { respectively. }\end{array}$ \\
\hline 6 & $\begin{array}{l}\text { Any pyrazinamide resistance is 6.95\% (Cl 6.07-7.91\%) and 8.77\% (7.53-10.13\%) among new and previously treated TB patients, } \\
\text { respectively. }\end{array}$ \\
\hline
\end{tabular}

in the current study. A major proportion of TB patients in India visit the private sector and the quality of TB care in the private sector has been shown to be suboptimal with wide variations in knowledge and adherence to guidelines, thereby increasing the risk of development of drug resistance ${ }^{3,4}$. Also, the survey only included patients with smear positive pulmonary $\mathrm{TB}$, excluding smearnegative pulmonary TB and extrapulmonary $\mathrm{TB}$, as well as patients from jails and prisons ${ }^{1,5}$. Among MDR TB patients, additional resistance to fluoroquinolones (Pre-XDR (PreExtensively Drug-Resistant TB) with fluoroquinolone resistance) was seen in $21.82 \%$ patients. This high proportion of resistance to fluoroquinolones necessitates that empirical use of fluoroquinolones in respiratory ailments should be discouraged. Also, previously it has been shown that high rates of treatment failure and deaths are associated with fluoroquinolone resistance in the Indian cohort of MDR-TB patients ${ }^{6}$. This finding limits the potential benefit of short course regime for MDR-TB patients which has currently been introduced in the country, as known resistance to fluoroquinolones is one of the exclusion criteria for the regime ${ }^{7}$. The proportion of INH resistance was $11.06 \%$ and $25.09 \%$, among new and previously treated TB patients, respectively. Considering this, the current recommendation of INH chemoprophylaxis for TB needs to be relooked at. It has been shown previously that aggressive community-wide IPT (INH Prophylaxis therapy) can have an impressive impact on reductions of drug-sensitive disease, but at the cost of increasing the selective pressure for resistance ${ }^{8}$. This finding is however in sync with recent recommendation to shift to treatment regimens for drug-sensitive TB where Ethambutol is a part of the regime throughout the treatment along with INH and Rifampicin, thus preventing possible amplification of Rifampicin resistance which could have happened in absence of Ethambutol in the regime ${ }^{9}$.

The individual state-wise data shows Maharashtra to have the highest proportion of MDR-TB in new TB patients $(7.72 \%)$, above than the national figure of $2.8 \%$. Similarly, Uttarakhand had the highest proportion of MDR TB in previously treated TB patients (20\%), above the national figure of $11.62 \%$. As per the survey, the distribution of MDR among the States that participated suggests areas for focus action. Strategies to map TB hotspots in the country are urgently required as they could be a possible causative factor for increased figures from a particular state even though the TB management in the rest of the state could be at par with the national standards. E.g. Previously the first case reports of TDR-TB (Total Drug Resistant TB) from India came from Mumbai in Maharashtra state and the current DRS survey shows Maharashtra as the state with highest proportion of MDR-TB in new TB patients ${ }^{1,10}$. The current survey showed that the highest proportion of primary MDR TB was in Maharashtra. It may be stated here that if India is the ground zero for global tuberculosis (TB) epidemic, then Mumbai, the capital of Maharashtra is the ground zero for India's epidemic of drug resistant TB $^{11}$. Mumbai has seen an increasing trend of drug resistant TB in the last two decades ${ }^{12}$. Mumbai accounts for the highest proportion of drug resistant TB patients in Maharashtra and recent reports indicate that the menace of drug-resistant TB grew by $36 \%$ in the city in last three years ${ }^{13}$. In a recent study from Western Maharashtra, the proportion of initial drug resistant TB was found to be an alarming $31.25 \%{ }^{14}$. In another study from Mumbai over an extended timeline (2005-2013), the authors found a six-fold increase in the absolute number of MDR-TB cases between the first 3-year period (2005-2007) and the last 3-year period (2011-2013), as well as the trend towards increasingly advanced patterns of resistance ${ }^{15}$. The relatively high rate of primary MDR-TB cases could be due to the identification of the undiagnosed resistance cases in the community. Thus, these findings may imply the need for intensifying active TB case finding using community-based health extension workers and this could help in timely identification of undiagnosed resistant strains $\mathrm{s}^{16}$. On the other hand, Uttarakhand had the highest proportion of secondary drug resistant TB patients. Secondary resistance may reflect poor implementation of Directly Observed Treatment Short course (DOTS) programme and should be taken as a serious challenge in the TB control programme ${ }^{16}$. Interestingly as per 
the state wise TB case notification data for 2017 it is evident that Uttarakhand accounted for highest proportion of TB patients who had a history of previous anti TB treatment, a factor which could be important in contributing to the high numbers of secondary drug resistant TB patients in the state $^{17}$.

Even though there was no age limitation, children (014 years) contributed $1.7 \%$ of the total population ${ }^{1}$. This proportion could have increased by usage of induced sputum, gastric aspirate and CBNAAT (Cartridge based Nucleic Acid Amplification Test) to define "microbiologically confirmed TB" as is currently being advocated in the recent RNTCP (Revised National Tuberculosis Control Programme) guidelines in India ${ }^{9}$. There was a low proportion of female TB patients $(27.99 \%)$ in the study ${ }^{1}$. This may highlight the fact that women are less likely to seek medical advice for early symptoms than males. This may be due to socio-cultural reasons associated with TBrelated stigmas, a factor which needs to be addressed with urgency ${ }^{18,19}$. The RNTCP needs to focus on raising the disease awareness in the female population. A notable limitation of the survey was absence of TB patients from the private sector ${ }^{1}$. It has been shown recently that about 2.2 million tuberculosis cases in India might have been treated in the private sector in 2014, which is two to three times higher than the current assumption ${ }^{3}$. This finding has revealed the hidden numbers, signifying the alarming magnitude of the problem unaccounted for in the official figures $^{20}$. These patients visiting the private sector are important as the standard of TB care in private sector in India still has a lot to be desired. It has been shown that factors for drug resistance were present in $68 \%$ of antiTB prescriptions from the private sector in India ${ }^{21}$. Apart from this, the lack of a reporting, monitoring or tracing mechanism of patients all these years has augmented the problem of absence of concrete data on drug resistance in the patients visiting the private sector. With compulsory notification of TB patients as mandated recently, these issues may have had their solution ${ }^{3}$. The inclusion of these TB patients visiting the private sector may present future NDRS surveys with a different result profile.

The key recommendations of the NDRS survey are: a. Setting up and strengthening drug resistance surveillance including using state of art next-generation sequencing. $b$. Moving rapidly towards universal DST and appropriately DST guided treatment. c. Strengthening epidemiologic intelligence for specific interventions based on local epidemiological profile ${ }^{1}$. These recommendations if followed with intent have the potential to positively change the present-day scenario of drug-resistant TB in India.

\section{Conclusion}

The findings of the NDRS survey are an eye opener for us. The future strategies in line with NSP (National Strategic Plan) (2017-2025) in India need to strongly consider the trends of drug resistance showcased by the survey. The capacity building of drug resistance diagnostics combined with compulsory notification of all TB patients may see future DRS surveys depicting an increased magnitude of the problem of drug-resistant TB in India and the policymakers should be aware of this possible trend in the coming years.

\section{References}

1. Ministry of Health and Family Welfare Gol. Report of the first national anti-tuberculosis drug resistance survey: India 2014-16, 2018. https://tbcindia.gov.in/showfile.php?lid=3315 (accessed April 9, 2018).

2. Global tuberculosis report 2018. Geneva: World Health Organization; 2018. Licence: CC BY-NC-SA 3.0 IGO.

3. Arinaminpathy N, Batra D, Khaparde S, et al. The number of privately treated tuberculosis cases in India: an estimation from drug sales data. Lancet Infect Dis. 2016; 16: 1255-60.

4. Satyanarayana S, Subbaraman R, Shete P, et al. Quality of tuberculosis care in India: a systematic review. Int J Tuberc Lung Dis. 2015; 19: 751-63.doi:10.5588/ijtld.15.0186

5. Chatterjee S, Poonawala H, Jain Y. Drug-resistant tuberculosis: is India ready for the challenge. BMJ Global Health. 2018; 3(4).

6. World Lung Conference 2010 - Association of poor culture conversion with fluoroquinolone resistance in Gujarat, Western India. R.N. Solanki, P V Dave, T V Bhalodia, S Chadha, F Wares, N Selvakumar, L S Chauhan, P Dewan.

7. Central TB Division, Ministry of Health and Family Welfare Programmatic Management of Drug Resistant TB (PMDT) in India. New Delhi: Directorate of General Health Services, Ministry of Health and Family Welfare, Government of India; 2017.

8. Mills HL, Cohen T, Colijn C. Community-wide isoniazid preventive therapy drives drug-resistant tuberculosis: a model-based analysis. Science translational medicine. 2013; 5(180): 180ra49-ra49.

9. Central TB Division, Ministry of Health and Family Welfare. Technical and operational guidelines for TB Control in India. New Delhi: Directorate of General Health Services, Ministry of Health and Family Welfare, Government of India; 2016.

10. Udwadia ZF, Amale RA, Ajbani KK, et al. Totally Drug-Resistant Tuberculosis in India. Clinical Infectious Diseases. 2012; 54(4): 57981.

11. Daftary A, Pai M. Tuberculosis therapy in Mumbai: Critical importance of drug-susceptibility testing. Lung India: Official Organ of Indian Chest Society. 2016; 33(3): 251-2.

12. Udwadia Z. Totally drug resistant-tuberculosis in India: The bad just got worse. The Journal of Association of Chest Physicians. 2016; 4(2): 41-2.

13. TNN. Drug- Resistant TB cases in Mumbai up 36\% in 3 years. Times of India [newspaper on the Internet]. 2018 Mar 24 [cited 2018 Aug 26]; Mumbai News. Available from: https://timesofindia.indiatimes.com/ city/mumbai/drug-resistant-tb-cases-in-mumbai-up-36-in-3-years/ articleshow/63436898.cms

14. More SW, Parande MA, Kamble SW, et al. Profile of drug-resistant tuberculosis in Western Maharashtra. Journal of Family Medicine and Primary Care. 2017; 6(1): 29-33.

15. Dalal A, Pawaskar A, Das M, et al. Resistance patterns among multidrug-resistant tuberculosis patients in greater metropolitan Mumbai: trends over time. PLoS One. 2015; 10(1): e0116798. 
16. Hamusse SD, Teshome D, Hussen MS, et al. Primary and secondary anti-tuberculosis drug resistance in Hitossa District of Arsi Zone, Oromia Regional State, Central Ethiopia. BMC Public Health. 2016; 16: 593.

17. Central TB Division, DGHS, MOHFW. India TB Report 2018 Revised National TB Control Programme, Annual Status Report. https:// tbcindia.gov.in/showfile.php?lid=3314 (accessed August 26, 2018).

18. Gosoniu GD, Ganapathy S, Kemp J, et al. Gender and socio-cultural determinants of delay to diagnosis of TB in Bangladesh, India and Malawi. The international journal of tuberculosis and lung disease: the official journal of the International Union against Tuberculosis and Lung Disease. 2008; 12(7): 848-55.

19. Samal J. Family perspectives in the care and support of tuberculosis patients: An Indian context. The Journal of Association of Chest Physicians. 2017; 5(2): 67-9.

20. Mishra G, Mulani J. Patients with tuberculosis in the private sector: counting the uncounted. The Lancet Infectious Diseases. 2017; 17(2): 134-5.

21. Mishra G, Mulani J. Tuberculosis prescription practices in private and public sector in India. Natl J Integr Res Med. 2013; 4(2): 71-78. 